\title{
Effects of Lateral Patellar Retinaculum Release for Recurrent Patella Dislocation: A Prospective
} Study

\author{
Xing-Liang Wang' \\ Chao Peng ${ }^{2}$ \\ You-Wei Tu ${ }^{2}$ \\ Yun-Peng Liu ${ }^{2}$ \\ Wei Zhang ${ }^{2}$ \\ Yan Zhang ${ }^{2}$ \\ Guo-Jun Hua ${ }^{3}$
}

'Department of Orthopedics, Wuxi Second People's Hospital, Wuxi, 2I4000, People's Republic of China; ${ }^{2}$ Department of Orthopedic Surgery, The No.904 Hospital of People's Liberation Army, Wuxi 2 I4000, People's Republic of China; ${ }^{3}$ Department of Orthopedic Surgery, Wuxi No.2 Chinese Medicine Hospital, Wuxi, 214000, People's Republic of China

Correspondence: Guo-Jun Hua

Department of Orthopedic Surgery, Wuxi No.2 Chinese Medicine Hospital, Wuxi, 214000, People's Republic of China Email hgjI306I@I63.com

Xing-Liang Wang

Department of Orthopedics, Wuxi

Second People's Hospital, Wuxi, 2I4000,

People's Republic of China

Email15006176567@I63.com
Purpose: Recurrent patellar dislocation (RPD) is the most common complication of patellar instability and the medial patellofemoral ligament (MPFL) reconstruction has become its reference treatment. Lateral patellar retinaculum (LPR) release used to be performed in association with MPFL reconstruction. The aim of this study was to investigate the added values of MPFL reconstruction plus LPR release for RPD.

Methods: After Institutional Review Board approval, RPD patients from October 2014 to April 2019 were randomly assigned into two groups (isolated MPFL reconstruction [Group I] and MPFL reconstruction plus LPR release [Group II]) and prospectively assessed until 12 months after surgery. Knee joints with flexion of $20^{\circ}$ were scanned by a 64-row CT scanner. Congruence angle (CA), patella tilt angle (PTA), lateral patellofemoral angle (LPFA), tibial tuberosity-trochlear groove distance and patellar tilt with the quadriceps relaxed and contracted were measured. Knee function was assessed by Lysholm knee score and International Knee Documentation Committee (IKDC) score. Patients were followed up for at least 12 months.

Results: A total of 87 RPD patients (45 for Group I and 42 for Group II) were selected in this study. Preoperative clinical characteristics were not significantly different across groups. No serious complications were noted in either group. It was statistically insignificant between the two group patients in terms of postoperative patella associated measurements $(\mathrm{P}>0.05$ for all). The Lysholm score and IKDC score of Group I $(84.5 \pm 7.1$ and $87.9 \pm 7.2)$ were significantly less than that of Group II $(89.7 \pm 8.7$ and $93.1 \pm 7.7)$, which indicated the better knee function of Group II.

Conclusion: LPR release plus MPFL reconstruction provides additional benefits compared with isolated MPFL reconstruction in knee function. A combination of surgical treatments for RPD should be recommended.

Keywords: recurrent patella dislocation, medial patellofemoral ligament reconstruction, lateral patellar retinaculum release, prospective, combination treatment

\section{Introduction}

Recurrent patella dislocation (RPD) is the most common complication of acute patellar dislocation that frequently causes symptoms such as joint pain, instability, interlocking, sense of dislocation and swelling. ${ }^{1}$ It was reported that conservative treatments for RPD patients are not adequate and surgical treatments are required. ${ }^{2,3}$ The medial patellofemoral ligament (MPFL) reconstruction has been recommended as the reference surgical treatment for RPD over the past decades. ${ }^{4,5}$ MPFL reconstruction is performed either in isolation or combined with other bony procedures due to the 
improved understanding of patellofemoral instability. ${ }^{6,7}$ On the other hand, the effect of isolated MPFL reconstruction can be lowered due to the preoperative patellar height, and the patellar was suggested to be drawn lower and deeper into the trochlear groove. ${ }^{8}$

The content of lateral patellar retinaculum (LPR) release is to release relatively tight lateral structures that can laterally pull the patella for balancing the patella in the trochlear groove. ${ }^{9}$ Since the isolated LPR release caused poor long-term results of RPD, the surgical procedure has no longer been considered as an isolated treatment. ${ }^{10}$ Several authors have reported great outcomes of MPFL reconstruction without associated lateral release. ${ }^{11,12}$ However, custom holds that LPR release in association with MPFL reconstruction is a nice combination strategy, although comparative trials are lacking. Du et al conducted a prospective study showing that MPFL reconstruction combined with LPR release is the best combination strategy among all combinations of surgical procedures. ${ }^{6}$ A prospective study with small sample size conducted by Malatray et al revealed that LPR release cannot provide added values to MPFL reconstruction for RPD. ${ }^{13}$ Therefore, it remains controversial whether the LPR release can provide added values to MPFL reconstruction for RPD patients.

The previous retrospective study conducted by Wang et al also demonstrated that MPFL reconstruction combined with LPR release is worthy to be promoted with the highest knee function scores. ${ }^{14}$ However, the comparison between isolated MPFL reconstruction and combined with LPR release was ignored. In addition, it is difficult to avoid the subjective bias due to the retrospective nature. The aim of this prospective study is to evaluate the effect of LPR release in association with MPFL reconstruction in order to provide high-level evidences for added values of LPR release. The hypothesis of this study is that the MPFL reconstruction plus LPR release is better than isolated MPFL reconstruction for RPD patients in terms of postoperative patella associated measurements and knee function.

\section{Patients and Methods}

This prospective study was approved by the institutional review board of the author's affiliated institutions (The No.904 Hospital of People's Liberation Army; Serial number: 201311164; date: 11/16/2013). Written informed consent was obtained from all participants. All procedures involving human participants were performed in accordance with the 1975 Helsinki declaration and its later amendments.

\section{Patients}

Patients with RPD between October 2014 and April 2019 were enrolled in this prospective comparative study according to the inclusion and exclusion criteria (Figure 1). Written informed consent was obtained from all participants who met the following criteria: (1) age between 18 and 45 years; (2) treatment-naïve when admitted to author's hospital; (3) diffuse pain and patellar instability at the knee joint with obvious tenderness above the medial femoral condyle; (4) RPD or symptoms of first patellar dislocation lasting over three months;. The exclusion criteria were (1) age less than 18 or more than 45 years; (2) received surgical treatments; (3) first patella dislocation; (4) generalized ligament laxity; (5) received treatment outside author's hospital; (6) any other diseases of the knee joint; (7) femoral trochlear dysplasia, which required osteotomy; (8) abnormal $\mathrm{Q}$ angles ${ }^{15}$ of the knee joints; (9) patellar dysplasia or patellar alta. The patients were informed of procedure information and possible complications of MPFL reconstruction in isolation or combined with LPR release. All enrolled patients provided written consent prior to enrolment in this study.

\section{Randomization}

The workflow is depicted in Figure 2. Randomization was performed by a simple computer program and each patient was numbered. Patients with odd numbers were allocated to isolated MPFL reconstruction (Group I) and others were allocated to MPFL reconstruction combined with LPR release (Group II) by the surgeons the day before the surgery. A researcher who was blinded to the surgery performed postoperative assessments of the patients. Patients were informed after surgery about the surgical procedure performed.

\section{Technique Details of MPFL Reconstruction}

All surgeries were performed by same group surgeons with at least 15-year experience in knee surgery. The schedule of MPFL reconstruction is shown in Figure 3 and Supplementary Figures 1-3. During MPFL reconstruction, an arthroscopic exploration was performed and subsequent chondral and meniscal pathology was undertaken. A bone tunnel was formed and enlarged behind the 


\section{Patients with RPD between October 2014 and April 2019 \\ $\mathrm{n}=168$}

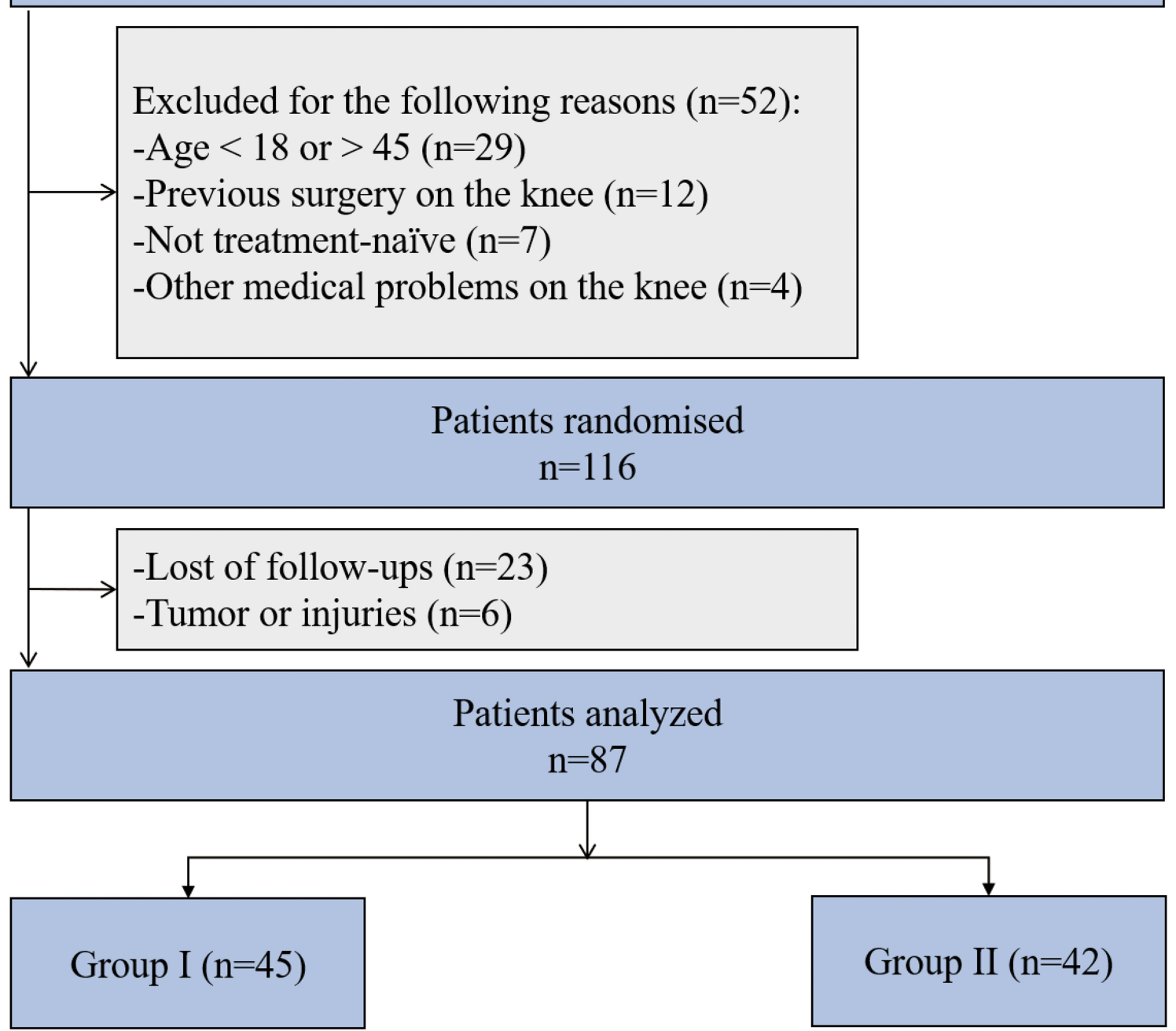

Figure I Consort diagram of the study.

Abbreviation: RPD, recurrent patella dislocation.

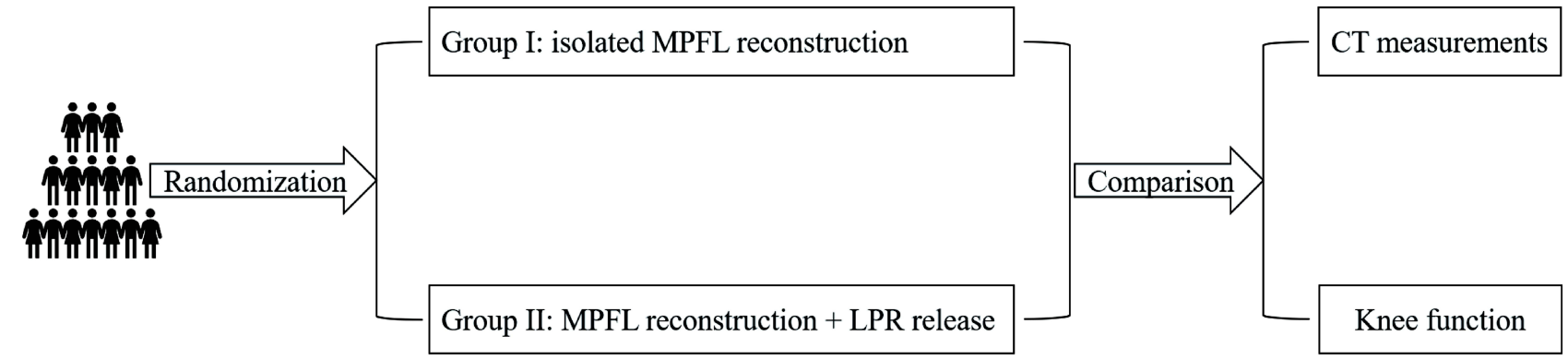

Figure 2 Workflow of necessary steps in this study. Randomization was performed and each patient was numbered. Patients with odd numbers were allocated to isolated MPFL reconstruction (Group I) and others were allocated to MPFL reconstruction combined with LPR release (Group II). Postoperative assessments including CT measurements and knee function were performed.

Abbreviations: CT, computed tomography; MPFL, medial patellofemoral ligaments; LPR, lateral patellar retinaculum. 

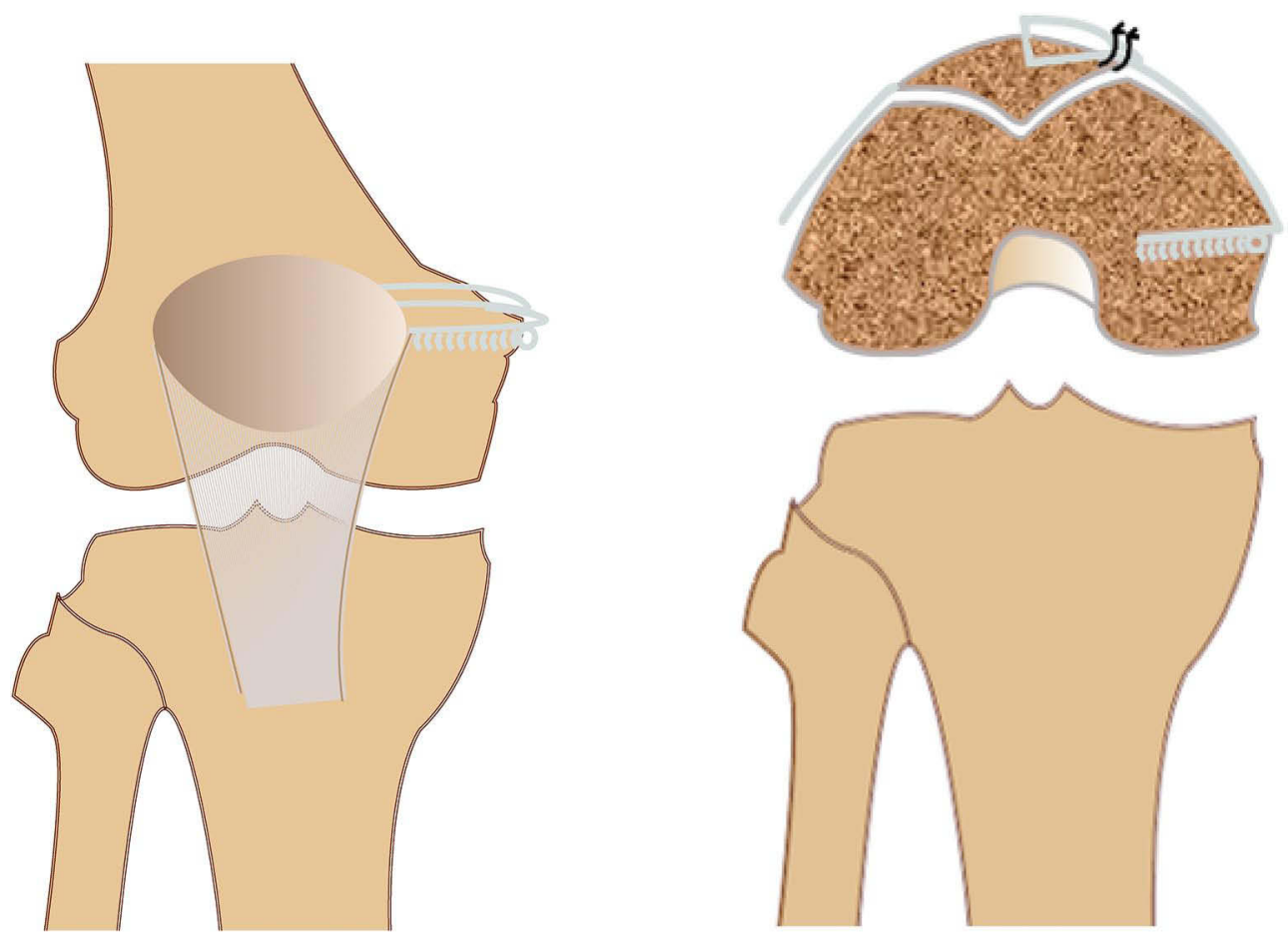

Figure 3 The schedule of MPFL reconstruction with autografts: femoral and patellar bone tunnels. Abbreviation: MPFL, medial patellofemoral ligaments.

highest point of the medial tuberosity of femur and the midpoint of the adductor tubercle. Autogenous semitendinosus tendons were used as autografts and prepared in a Y-shape to be fixed on the femoral tunnel with a bioresorbable screw. The tension of tendon was adjusted under the arthroscope by pulling the braided suture on bilateral sides of the tendon. The joint congruence and motion track of the patella returned to normal.

\section{Technique Details of LPR Release}

The scheme of LPR release (Figure 4 and Supplementary Figures 4-6) is relatively simple and performed prior to MPFL reconstruction during the arthroscopic exploration in order that the tension of the MPFL graft can be well controlled. ${ }^{16}$ The tense fibrous structure at the lateral aspect of the patella was cut and the LPR was released to the lower and lateral borders of the patella. The vastus lateralis oblique muscle was partially cut simultaneously to avoid adhesion.

\section{Postoperative Rehabilitation}

All patients in this study experienced the unified postoperative rehabilitation including ice compress, ankle pump exercise and a series of range of motion (ROM) exercise. Ice compressions can be performed as soon as possible with no more than 15 minutes each time and the interval of two ice compresses on the same area should be at least 2 hours. The purpose of the ankle pump exercise was to contract the calf muscles through the up and down movement of the ankle joint, reduce venous stasis and relieve the swelling of the lower limbs. ROM exercise for these patients mainly included knee extension and bend.

\section{Data Collection and Follow-Up}

All knee joints were scanned at $20^{\circ}$ flexion by computed tomography (CT). Preoperatively, measurements of the congruence angle (CA), ${ }^{17}$ patellar tilt angle (PTA) ${ }^{18}$ lateral patellofemoral angle (LPFA) ${ }^{19}$ and tibial tuberositytrochlear groove (TT-TG) distance were performed on each patient using $\mathrm{CT}$ three-dimensional reconstruction techniques. CT scans have the ability to directly measure the CA, PTA, LPFA at the mid-patellar level. Lysholm knee score $^{20}$ and subjective International Knee Documentation Committee (IKDC) score ${ }^{21}$ were subsequently used to evaluate knee joint function. The Lysholm score focuses on knee instability and the IKDC score emphasizes the evaluation of knee function recovery.

All patients were followed up for at least 12 months. The follow-up contents included CT measurements and 

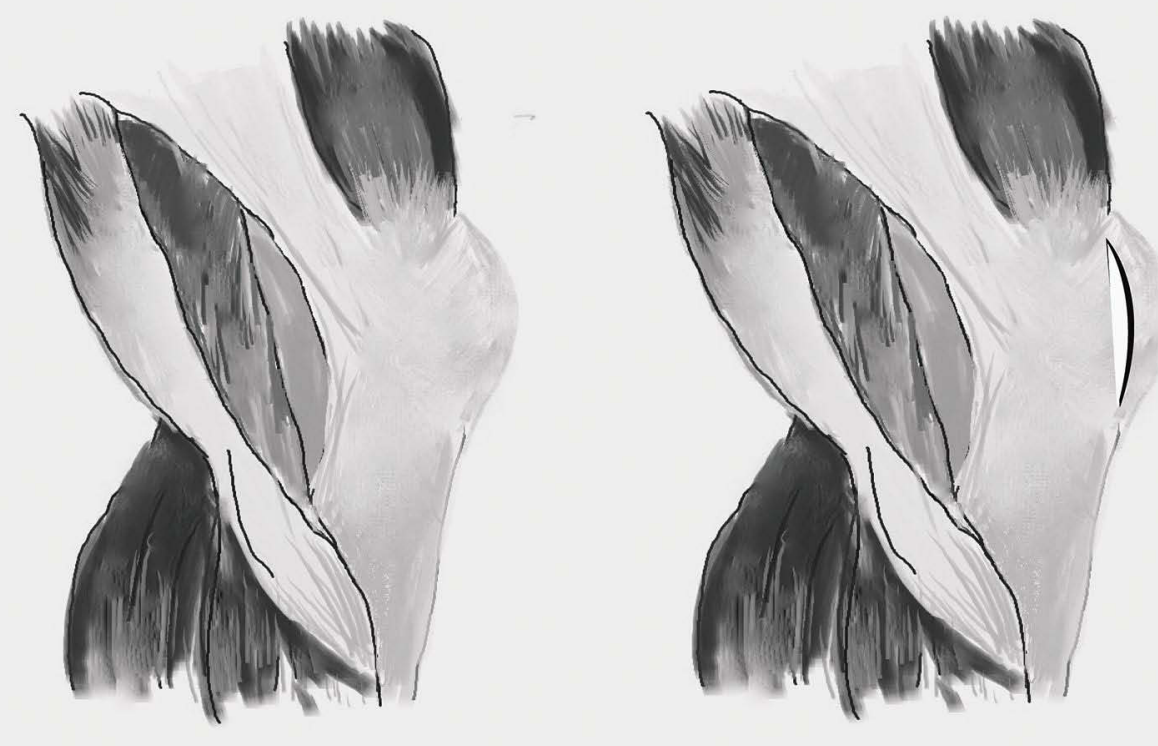

Figure 4 The schedule of LPR release.

Abbreviation: LPR, lateral patellar retinaculum.

knee function evaluations. Patellar tilt (PT) was evaluated with the quadriceps relaxed (PTQR) and contracted (PTQC). At 12 months postoperative patients underwent the same standard CT scan and completed Lysholm and IKDC score forms.

\section{Sample Size Calculation}

The sample size was calculated using PASS software (Power Analysis and Sample size, version 15.0.5). The calculation was done based on the assumption that a type I error probability of 0.05 and power of 0.9 . From a previous study, ${ }^{14}$ the standard deviation of Lysholm knee scores was 4.64 and 5.99, and a difference in Lysholm scores between two groups was 3.82. Then, the estimated sample size was 86 .

\section{Statistical Analysis}

All statistical analyses were performed using SPSS (version 19.0; Chicago, IL, USA). Results are presented as mean $\pm \mathrm{SD}$. The sample distribution was normal and homoscedastic. Student's $t$-test was used for quantitative data (CA, PTA, LPFA, TT-TG distance, PTQR, PTQC and knee function scores) between two groups. Chi-squared test or Fisher's exact test was used for qualitative data. A two-sided $P<0.05$ was considered significant.

\section{Results}

\section{Baseline Characteristics}

A total of 168 patients with RPD were admitted to author's hospital. According to the inclusion and exclusion criteria, 116 patients were finally included into this study. Twentythree of 116 (19.8\%) patients were lost of follow-up and 6 were unable to complete follow-up because of medical reasons (tumor (3) and injuries (3)). Final analysis was performed on 87 of $116(75 \%)$ patients, with 45 in the Group I and 42 in the Group II (Figure 1). There were no statistically significant differences in baseline characteristics between these two groups ( $P>0.05$ for all) (Table 1$)$.

\section{Preoperative CT Measurement and Knee Function Results}

As is shown in Table 2, no significant differences were found in preoperative CT measurement and knee function results including congruence angle (31.87 \pm 4.23 and $32.64 \pm 3.91$, $P=0.381)$, patellar tilt angle $(13.29 \pm 2.17$ and $13.47 \pm 2.23$, $P=0.704)$, lateral patellofemoral angle $(-2.6 \pm 3.8$ and $-2.4 \pm$ 4.7, $P=0.827)$, Lysholm score $(55.4 \pm 5.1$ and $56.7 \pm 6.8$, $P=0.314)$, and IKDC score $(41.2 \pm 5.7$ and $42.6 \pm 5.3$, $P=0.240$ ), respectively. Therefore, we could suppose that all preoperative informations (Tables 1 and 2) of these two group patients are similar allowing for comparison in this study. 
Table I Demographic Characteristics of the Population of the Study

\begin{tabular}{|l|l|l|l|}
\hline Variables & $\begin{array}{l}\text { Group } \\
\text { I (n=45) }\end{array}$ & $\begin{array}{l}\text { Group II } \\
(\mathbf{n}=\mathbf{4 2})\end{array}$ & $\boldsymbol{P}$ value \\
\hline Age (years) & $24.9 \pm 7.2$ & $27.1 \pm 8.9$ & 0.207 \\
Males/females & $17 / 28$ & $20 / 22$ & 0.354 \\
BMI & $22 \pm 7.9$ & $24 \pm 7.2$ & 0.222 \\
Left side/right side & $21 / 24$ & $23 / 19$ & 0.450 \\
Duration of dislocation & $18.1 \pm 6.7$ & $19.8 \pm 7.2$ & 0.257 \\
(months) & & & \\
Number of dislocations & $4.1 \pm 2.1$ & $3.7 \pm 1.9$ & 0.355 \\
\hline
\end{tabular}

Notes: Group I: isolated MPFL reconstruction; Group II: MPFL reconstruction combined with LPR release.

Abbreviations: BMI, body mass index; MPFL, medial patellofemoral ligament; LPR, lateral patellar retinaculum.

Table 2 Comparisons of Preoperative Patella Associated Measurements and Knee Function Between the Two Groups

\begin{tabular}{|l|l|l|l|}
\hline Variables & $\begin{array}{l}\text { Group } \\
\text { I }(\mathbf{n = 4 5 )}\end{array}$ & $\begin{array}{l}\text { Group II } \\
(\mathbf{n = 4 2 )}\end{array}$ & $\boldsymbol{P}$ value \\
\hline Congruence angle & $31.87 \pm 4.23$ & $32.64 \pm 3.91$ & $0.38 \mathrm{I}$ \\
Patellar tilt angle & $13.29 \pm 2.17$ & $13.47 \pm 2.23$ & 0.704 \\
Lateral patellofemoral & $-2.6 \pm 3.8$ & $-2.4 \pm 4.7$ & 0.827 \\
angle & & & \\
TT-TG distance (mm) & $16.7 \pm 2.9$ & $17.4 \pm 3.2$ & 0.288 \\
Lysholm score & $55.4 \pm 5.1$ & $56.7 \pm 6.8$ & 0.314 \\
IKDC score & $41.2 \pm 5.7$ & $42.6 \pm 5.3$ & 0.240 \\
\hline
\end{tabular}

Notes: Group I: isolated MPFL reconstruction; Group II: MPFL reconstruction combined with LPR release.

Abbreviations: MPFL, medial patellofemoral ligament; LPR, lateral patellar retinaculum; IKDC, International Knee Documentation Committee; TT-TG, tibial tuberosity-trochlear groove.

\section{Postoperative Assessment and Follow-Up}

Except 29 patients that were lost to follow-up, other patients were followed up for at least 12 months postoperatively. No serious complications were noted in either group. Postoperative 12th-month CT measurements (Table 3) returned to a similar range in both groups and were statistically insignificant in congruence angle (15.6 \pm 4.2 and $15.9 \pm 5.8, P=0.782)$, patellar tilt angle $(7.21 \pm$ 1.45 and $7.38 \pm 1.57, P=0.601)$, lateral patellar tilt angle (8.6 \pm 3.1 and $7.8 \pm 3.9, P=0.291)$, PTQR $(22.4 \pm 6.2$ and $21.7 \pm 6.8, P=0.617)$ and PTQC $(27.8 \pm 7.4$ and $25.6 \pm 8.1$, $P=0.189$ ). For postoperative 12th-month knee function evaluation (Table 3 ), it was statistically significant in Lysholm score ( $84.5 \pm 7.1$ and $89.7 \pm 8.7, P=0.003)$, and IKDC score $(87.9 \pm 7.2$ and $93.1 \pm 7.7, P=0.002)$, indicating more clinical benefits were provided by MPFL reconstruction plus LPR release in Group II.
Table 3 Comparison of Postoperative 12th-Month Patella Associated Measurements and Knee Function Between Two Groups

\begin{tabular}{|l|l|l|l|}
\hline Variables & $\begin{array}{l}\text { Group } \\
\text { I (n=45) }\end{array}$ & $\begin{array}{l}\text { Group II } \\
(\mathbf{n = 4 2 )}\end{array}$ & P value \\
\hline Congruence angle & $15.6 \pm 4.2$ & $15.9 \pm 5.8$ & 0.782 \\
Patellar tilt angle & $7.21 \pm 1.45$ & $7.38 \pm 1.57$ & 0.601 \\
Lateral patellofemoral & $8.6 \pm 3.1$ & $7.8 \pm 3.9$ & 0.291 \\
angle & & & \\
Postoperative PTQR & $22.4 \pm 6.2$ & $21.7 \pm 6.8$ & 0.617 \\
Postoperative PTQC & $27.8 \pm 7.4$ & $25.6 \pm 8.1$ & 0.189 \\
Lysholm score & $84.5 \pm 7.1$ & $89.7 \pm 8.7$ & 0.003 \\
IKDC score & $87.9 \pm 7.2$ & $93.1 \pm 7.7$ & 0.002 \\
\hline
\end{tabular}

Notes: Group I: isolated MPFL reconstruction; Group II: MPFL reconstruction combined with LPR release.

Abbreviations: MPFL, medial patellofemoral ligament; LPR, lateral patellar retinaculum; IKDC, International Knee Documentation Committee; PTQR, patellar tilt with the quadriceps relaxed; PTQC, patellar tilt with the quadriceps contracted.

\section{Discussion}

To our knowledge, this is the first prospective study showing that, post-operatively, the MPFL reconstruction and LPR release had the higher Lysholm knee and IKDC score. The LPR release can provide added values that can aid in knee function recovery for RPD patients.

The surgical treatment strategies, especially for RPD patients, are always controversial. It was reported that RPD occurs to the lateral side leading to ruptures of the MPFL in almost $90 \%$ of all cases. ${ }^{22,23}$ Tsubosaka et $\mathrm{al}^{24}$ demonstrated that MPFL is the predominant soft tissue in preventing patella dislocation and maintaining appropriate patellar tracking. Therefore, it is no doubt that MPFL reconstruction becomes as the reference for the basic treatment of RPD. ${ }^{22}$ In the past, MPFL reconstruction, LPR release and medial patellar retinaculum (MPR) plication were proposed as three basic surgical methods for correcting patellar movement and instability. ${ }^{6}$ The combination strategies of these surgical methods were frequently proposed over the past years. ${ }^{6,14,25,26} \mathrm{Du}$ et $\mathrm{al}^{6}$ conducted a prospective study showing MPFL reconstruction in association with LPR release is a best combination surgical strategy in terms of postoperative patellar stability and knee joint function. Zhao et $\mathrm{al}^{27}$ demonstrated MPFL reconstruction is more effective than MPR plication in combination surgeries including LPR release, which implied LPR release plays an indispensable role in traditional combination surgical treatments. However, several researchers have reported that MPFL reconstruction in isolation could also improve RPD patients' quality of 
life. ${ }^{27,28}$ Moreover, Malatray et $\mathrm{al}^{13}$ proposed that LPR release is not recommended in association with MPFL reconstruction for the treatment of RPD. The limited sample size (only 33 patients) of the study conducted by Malatray et al can lead to a risk of missing a significant difference between the two groups. Considering this, our prospective study included more patients for analysis. Recent studies also investigated the effect of MPFL reconstruction in isolation for patellar instability and influence factors. ${ }^{8,29}$ MPFL reconstruction decreases patellar height ratio and the preoperative patellar height affects the outcome of the surgery. The higher the preoperative patellar height, the greater was the lowering effect of MPFL reconstruction. And thus, drawing the patella lower and deeper into the trochlear groove may improve patellar tracking for greater knee function.

No significant differences were observed in terms of patellar stability between isolated MPFL reconstruction group and MPFL reconstruction plus LPR release group. However, MPFL reconstruction combined with LPR release can provide more clinical benefits to knee function recovery. Studies conducted by Du et al or Wang et al did not include isolated MPFL reconstruction for comparisons. ${ }^{6,14}$ Abovementioned findings of this prospective study can provide further evidence for them. We supposed that the combined procedure (MPFL reconstruction plus LPR release) can make the patella more automatically match the anatomical structure of the trochlear groove, leading to the more natural movements. If the LPR release is not additionally performed, the movement of the patella is subjected to forces in the opposite direction because of MPFL and LPR, which might potentially affect the knee function.

The study is prospective with rational sample size which can provide solid evidence. However, we also realized some limitations. The major defect of this study is that the followup time of 12 months is not enough to detect more long-term differences between these two groups. Furthermore, this study is just at the single-center level of which the results might not be very representative. Therefore, a big-sample, multi-center study is required for further investigation in the future. At this present, we consider that whether LPR release should be in association with MPFL reconstruction also depends on the operation time, postoperative complication and patient's physical condition. Further studies should also detailedly record complications and more clinical related data.
In conclusion, better outcomes of knee function recovery which were provided by LPR release in association with MPFL reconstruction indicated that LPR release combined with MPFL reconstruction should continue to be proposed. In order to obtain better clinical results, it is worthwhile to generalize the combination of MPFL reconstruction and LPR release for RPD patients.

\section{Abbreviations}

$\mathrm{CA}$, congruence angle; CT, computed tomography; IKDC, International Knee Documentation Committee; LPFA, lateral patellofemoral angle; LPR, lateral patellar retinaculum; MPFL, medial patellofemoral ligament; PTA, patellar tilt angle; RPD, recurrent patella dislocation.

\section{Data Sharing Statement}

The datasets analyzed during the current study are available from the corresponding authors on reasonable request.

\section{Ethical Statement}

This prospective study was approved by the institutional review board of the author's affiliated institutions (The No.904 Hospital of People's Liberation Army; Serial number: 201311164; date: 11/16/2013).

\section{Acknowledgments}

We thank Bruce Zhang, PhD, from Department of Economics College of Arts and Sciences, University at Buffalo 415 Fronczak Hall, North Campus Buffalo, for editing the language.

\section{Author Contributions}

All authors made a significant contribution to the work reported, whether that is in the conception, study design, execution, acquisition of data, analysis and interpretation, or in all these areas; took part in drafting, revising or critically reviewing the article; gave final approval of the version to be published; have agreed on the journal to which the article has been submitted; and agree to be accountable for all aspects of the work.

\section{Funding}

This research was supported by the youth program of Wuxi Municipal Health Planning Commission (Q201772) and the medicine and public health technology program of Wuxi science and technology development (CSE31N 1618). 


\section{Disclosure}

The authors report no conflicts of interest in this work.

\section{References}

1. Colvin AC, West RV. Patellar instability. J Bone Joint Surg Am. 2008;90(12):2751-2762. doi:10.2106/JBJS.H.00211

2. Fithian DC, Paxton EW, Cohen AB. Indications in the treatment of patellar instability. J Knee Surg. 2004;17:47-56. doi:10.1055/s-00301247149

3. Dannawi Z, Khanduja V, Palmer CR, El-Zebdeh M. Evaluation of the modified Elmslie-Trillat procedure for patellofemoral dysfunction. Orthopedics. 2010;33:13.

4. Panagopoulos A, van Niekerk L, Triantafillopoulos IK. MPFL reconstruction for recurrent patella dislocation: a new surgical technique and review of the literature. Int J Sports Med. 2008;29:359-365. doi:10.1055/s-2007-965360

5. Bouras T, U E, Brown A, Gallacher P, Barnett A. Isolated medial patellofemoral ligament reconstruction significantly improved quality of life in patients with recurrent patella dislocation. Knee Surg Sports Traumatol Arthrosc. 2019;27(11):3513-3517. doi:10.1007/s00167-01905447-w

6. Du H, Tian XX, Guo FQ, et al. Evaluation of different surgical methods in treating recurrent patella dislocation after three-dimensional reconstruction. Int Orthop. 2017;41:2517-2524. doi:10.1007/s00264-017-3552-9

7. Becker R, Hirschmann MT, Karlsson J. The complexity of patellofemoral instability. Knee Surg Sports Traumatol Arthrosc. 2018;26: 675-676. doi:10.1007/s00167-018-4860-3

8. Luceri F, Roger J, Randelli PS, Lustig S, Servien E. How does isolated medial patellofemoral ligament reconstruction influence patellar height? Am J Sports Med. 2020;48(4):895-900. doi:10.11 77/0363546520902132

9. Kolowich PA, Paulos LE, Rosenberg TD, Farnsworth S. Lateral release of the patella: indications and contraindications. $\mathrm{Am}$ J Sports Med. 1990;18:359-365. doi:10.1177/036354659001800 405

10. Lattermann C, Toth J, Bach BR Jr. The role of lateral retinacular release in the treatment of patellar instability. Sports Med Arthrosc Rev. 2007;15:57-60. doi:10.1097/JSA.0b013e318042af30

11. Zhang QC, Wang SF, Fu XS. Arthroscopic medial patellofemoral ligament reconstruction combined with lateral retinacular release for recurrent patellar dislocation. Zhongguo Gu Shang. 2015;28: 599-602.

12. Zhang H, Hong L, Geng X, Wang X, Feng H. Reconstruction of medial patellofemoral ligament for recurrent patellar dislocation. Zhongguo Xiu Fu Chong Jian Wai Ke Za Zhi. 2011;25:925-930.

13. Malatray M, Magnussen R, Lustig S, Servien E. Lateral retinacular release is not recommended in association to MPFL reconstruction in recurrent patellar dislocation. Knee Surg Sports Traumatol Arthrosc. 2019;27:2659-2664. doi:10.1007/s00167-018-5294-7

14. Wang XL, Wang XL, Peng C, Yang JJ, Hua GJ, Liu YP. Comparative study of three different combination surgical methods for recurrent patella dislocation. J Orthop Sci. 2020;25:1067-1071. doi:10.1016/j. jos.2020.01.008

15. Paranjape S, Singhania N. Effect of body positions on quadriceps angle measurement. SciMed J. 2019;1:20-24. doi:10.28991/SciMedJ2019-0101-3
16. Christoforakis J, Bull AM, Strachan RK, Shymkiw R, Senavongse W, Amis AA. Effects of lateral retinacular release on the lateral stability of the patella. Knee Surg Sports Traumatol Arthrosc. 2006;14: 273-277. doi:10.1007/s00167-005-0699-5

17. Merchant AC, Mercer RL, Jacobsen RH, Cool CR. Roentgenographic analysis of patellofemoral congruence. J Bone Joint Surg Am. 1974;56:1391-1396. doi:10.2106/00004623-197456070-00007

18. Schutzer SF, Ramsby GR, Fulkerson JP. The evaluation of patellofemoral pain using computerized tomography. A preliminary study. Clin Orthop Relat Res. 1986;204:286-293.

19. Laurin CA, Lévesque HP, Dussault R, Labelle H, Peides JP. The abnormal lateral patellofemoral angle: a diagnostic roentgenographic sign of recurrent patellar subluxation. J Bone Joint Surg Am. 1978;60:55-60. doi:10.2106/00004623-197860010-00007

20. Lysholm J, Gillquist J. Evaluation of knee ligament surgery results with special emphasis on use of a scoring scale. Am J Sports Med. 1982;10:150-154. doi:10.1177/036354658201000306

21. Irrgang JJ, Anderson AF, Boland AL, et al. Development and validation of the international knee documentation committee subjective knee form. Am J Sports Med. 2001;29:600-613. doi:10.1177/ 03635465010290051301

22. Balcarek P, Walde TA, Frosch S, et al. Patellar dislocations in children, adolescents and adults: a comparative MRI study of medial patellofemoral ligament injury patterns and trochlear groove anatomy. Eur J Radiol. 2011;79:415-420. doi:10.1016/j.ejrad.2010.06.042

23. Balcarek P, Jung K, Ammon J, et al. Anatomy of lateral patellar instability: trochlear dysplasia and tibial tubercle-trochlear groove distance is more pronounced in women who dislocate the patella. $\mathrm{Am}$ J Sports Med. 2010;38:2320-2327. doi:10.1177/0363546510373887

24. Tsubosaka M, Muratsu H, Takayama K, Miya H, Kuroda R, Matsumoto T. Comparison of intraoperative soft tissue balance between cruciate-retaining and posterior-stabilized total knee arthroplasty performed by a newly developed medial preserving gap technique. J Arthroplasty. 2018;33:729-734. doi:10.1016/j.arth.2017.09.070

25. Wegmann H, Würnschimmel C, Kraus T, et al. Medial patellofemoral ligament (MPFL) reconstruction in combination with a modified grammont technique leads to favorable mid-term results in adolescents with recurrent patellofemoral dislocations. Knee Surg Sports Traumatol Arthrosc. 2018;26:705-709. doi:10.1007/s00167-017-4425-x

26. Damasena I, Blythe M, Wysocki D, Kelly D, Annear P. Medial patellofemoral ligament reconstruction combined with distal realignment for recurrent dislocations of the patella: 5-year results of a randomized controlled trial. Am J Sports Med. 2017;45 (2):369-376. doi:10.1177/0363546516666352

27. Zhao J, Huangfu X, He Y. The role of medial retinaculum plication versus medial patellofemoral ligament reconstruction in combined procedures for recurrent patellar instability in adults. Am J Sports Med. 2012;40(6):1355-1364. doi:10.1177/0363546512439193

28. Schneider DK, Grawe B, Magnussen RA, et al. Outcomes after isolated medial patellofemoral ligament reconstruction for the treatment of recurrent lateral patellar dislocations: a systematic review and meta-analysis. Am J Sports Med. 2016;44(11):2993-3005. doi:10.1177/0363546515624673

29. Hiemstra LA, Kerslake S, Lafave MR, Tucker A. Patella alta is reduced following MPFL reconstruction but has no effect on quality-of-life outcomes in patients with patellofemoral instability. Knee Surg Sports Traumatol Arthrosc. 2021;29(2):546-552. doi:10. 1007/s00167-020-05977-8 


\section{Publish your work in this journal}

The International Journal of General Medicine is an international, peer-reviewed open-access journal that focuses on general and internal medicine, pathogenesis, epidemiology, diagnosis, monitoring and treatment protocols. The journal is characterized by the rapid reporting of reviews, original research and clinical studies across all disease areas. The manuscript management system is completely online and includes a very quick and fair peer-review system, which is all easy to use. Visit http://www.dovepress.com/ testimonials.php to read real quotes from published authors.

Submit your manuscript here: https://www.dovepress.com/international-journal-of-general-medicine-journal 